\title{
EDUCATIONAND TRAINING Evaluating the impact of teaching sessions on NHS values to foundation doctors: a thematic analysis of participants' reflections
}

\author{
Authors: Linda Watkins, ${ }^{A}$ Sarita Sochart ${ }^{B}$ and Paul Baker ${ }^{C}$
}

\section{Background}

The Francis report highlighted the role of culture and values within the NHS. How to effectively teach the values of the NHS constitution remains unanswered. We evaluated NHS values training delivered to foundation doctors.

Methods

Standardised training was delivered to foundation doctors throughout the school. Post-session reflective forms were voluntarily completed by participants. Reflections over a 2-year period were analysed and thematic qualitative analysis performed.

Results

One-hundred and thirty-one reflections were analysed. Common words included patients, caring and communication. Many themes were patient focused including 'advocating for patients / patient-centred care', 'patient safety' and 'small things = big difference'. Other themes were directly linked to specific values presented (eg 'dignity', 'teamwork' and 'compassion/empathy') or related to personal development (eg 'encouraging and improving reflection' and 'changing own practice').

Conclusion

Patient focus and a spectrum of themes were demonstrated; this session can address a variety of values and produce key lessons in learners.

KEYWORDS: NHS values, values-based teaching, Francis report, postgraduate medical education, ethical erosion

DOI: $10.7861 /$ fhj.2020-0055

\section{Introduction}

The publication of the Francis report following the MidStaffordshire NHS Foundation Trust public inquiry highlighted

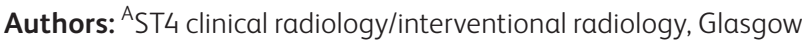
Royal Infirmary, Glasgow, UK; ${ }^{\text {B }}$ consultant stroke physician and foundation / academic foundation programme director, Pennine Acute Hospitals NHS Trust, Greater Manchester, UK; ${ }^{\text {C }}$ deputy postgraduate dean, Health Education England (North West), Manchester, UK that the culture within our hospitals strongly dictates the patient experience. One key lesson was that there should be 'emphasis on and commitment to common values throughout the system by all within it'. The NHS constitution puts forward principles that guide the operation and functioning of the NHS and states that these are underpinned by six NHS values (Box 1). ${ }^{2}$

While these may seem largely common sense, the phenomenon of ethical erosion, where multiple small compromises or increasing cynicism over a career lead to doctors becoming less ethical, is well-recognised enough to appear in the Oxford Concise Medical Dictionary. ${ }^{3,4}$

If values are to be used to inform or make decisions in the NHS, it is fundamental that we are all working with the same values in mind. ${ }^{5}$ We therefore must all be aware of what the NHS values are and consider how these are relevant to our role. The government's own response to the Francis report highlighted the necessity of education and training in achieving cultural change within the NHS. ${ }^{6}$ A follow-up paper again highlighted the need for staff training and ensuring that new recruits represent the values of the NHS. ${ }^{7}$ Despite all of this, little has been written around the actual delivery of education and training on values, particularly within the NHS. How do we effectively deliver values-based teaching?

Here, we present the results of a thematic analysis of participants' reflections following training sessions on NHS values.

\section{Methods}

Teaching on NHS values was delivered to doctors in foundation years as part of their mandatory teaching. The package was developed with the training provider 'What If... Coaching'. Sessions comprised an introduction, expectations and aspirations for the session, information on facts and figures of the NHS, why organisations have values and mission statements and why they are important, group work looking at complaints cases, and a

\section{Box 1. NHS values}

Working together for patients.

Respect and dignity.

Commitment to quality of care.

Compassion.

Improving lives.

Everyone counts. 


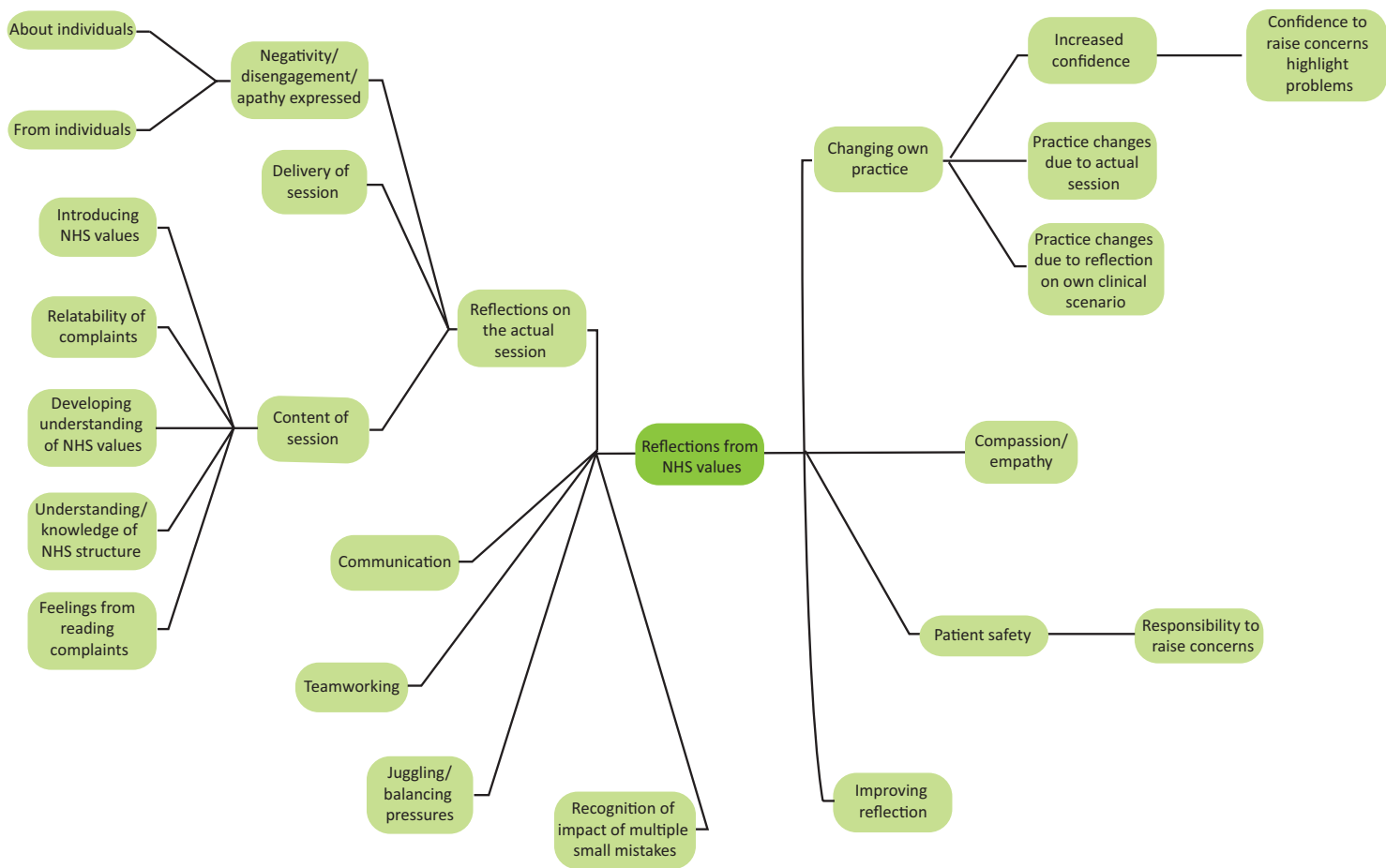

Fig 1. Initial themes identified from a read-through of data from portfolio reflections.

plenary where the groups gave feedback from their discussions before the session was concluded with revisiting the expectations and aspirations and time for questions. An example complaint letter for the group work component is included in supplementary material S1. This has been altered to maintain anonymity.

Participants were advised that there was a specific reflection form on their ePortfolio that could be used to reflect on NHS values and used to evidence coverage of various aspects of the foundation curriculum. The online form used Gibbs reflective cycle to lead participants through their reflections. ${ }^{8}$ Reflections could be specific to the teaching session or about a situation encountered in their clinical practice. All Horus users gave consent for anonymous data to be used for audit and research. Portfolio reflections over 2 years were analysed using qualitative methods. Initial themes were identified by the authors and are outlined in Fig 1. Further analysis was aided by qualitative analysis software, and themes were condensed and others added as a result (Fig 2). Reflections were mapped to themes and the results are presented.

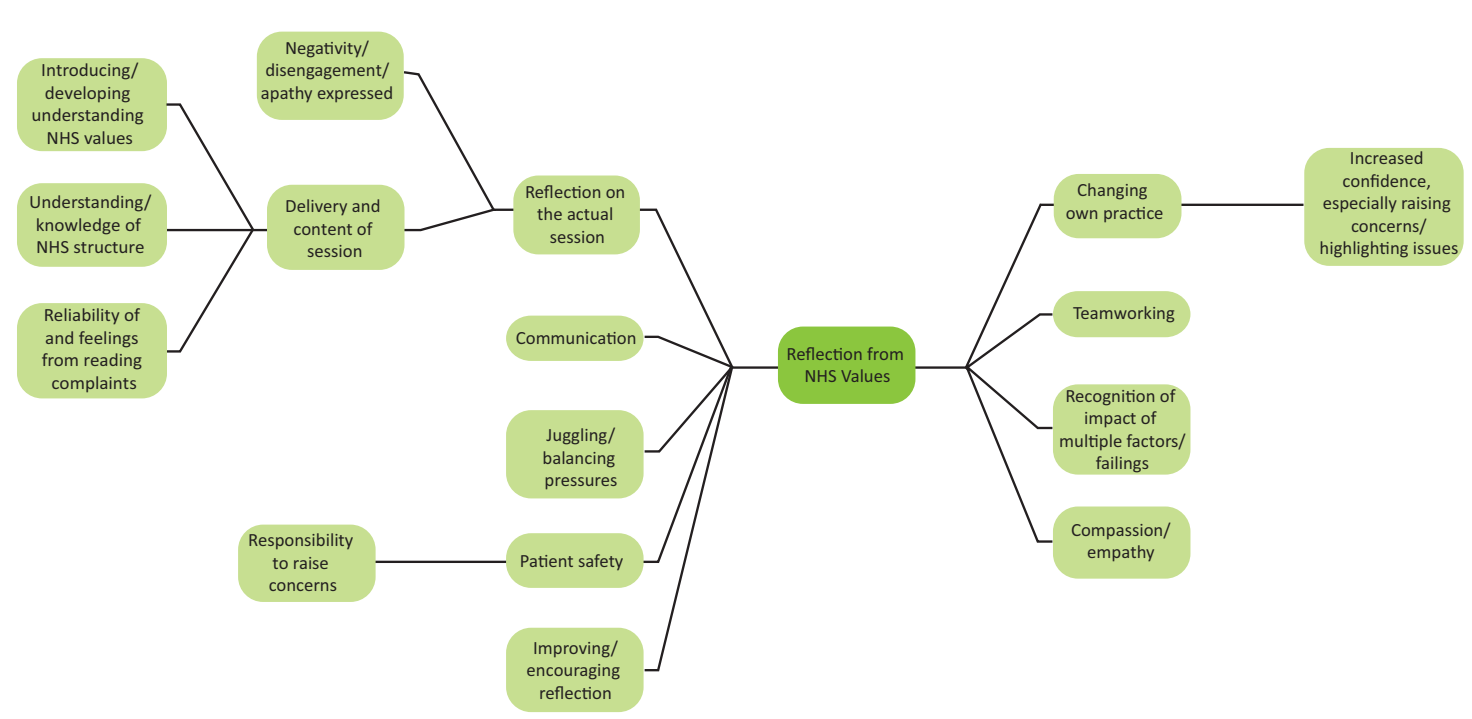

Fig 2. Condensing themes to allow structured thematical analysis. Three additional ideas (dignity, advocating for patients and small things $=$ big difference) represent themes identified through further analysis using qualitative research software. 
Table 1. Top ten words in the submitted reflections

\begin{tabular}{|c|c|c|c|}
\hline Word & Count & $\begin{array}{l}\text { Weighted } \\
\text { percentage }\end{array}$ & Similar words \\
\hline patients' & 910 & 4.22 & $\begin{array}{l}\text { patient, 'patient, } \\
\text { patients }\end{array}$ \\
\hline values & 458 & 2.13 & $\begin{array}{l}\text { value, valued, values', } \\
\text { valuing }\end{array}$ \\
\hline NHS & 430 & 2.00 & NHS' \\
\hline caring & 277 & 1.29 & care, care', cared \\
\hline session & 201 & 0.93 & sessions \\
\hline discussion & 193 & 0.90 & $\begin{array}{l}\text { discuss, discussed, } \\
\text { discusses, discussing, } \\
\text { discussions }\end{array}$ \\
\hline case & 163 & 0.76 & cases \\
\hline works & 163 & 0.76 & $\begin{array}{l}\text { work, worked, working, } \\
\text { 'working }\end{array}$ \\
\hline family & 162 & 0.75 & families \\
\hline communication & 160 & 0.74 & $\begin{array}{l}\text { communicate, } \\
\text { communicated, } \\
\text { communicating, } \\
\text { communications, } \\
\text { communicative, } \\
\text { communities, } \\
\text { community }\end{array}$ \\
\hline
\end{tabular}

\section{Results}

One-hundred and thirty-one reflections were analysed. The most common words found in the reflections are shown in Table 1 and Fig 3. These both illustrate the focus on patients that was apparent in almost every single reflection.

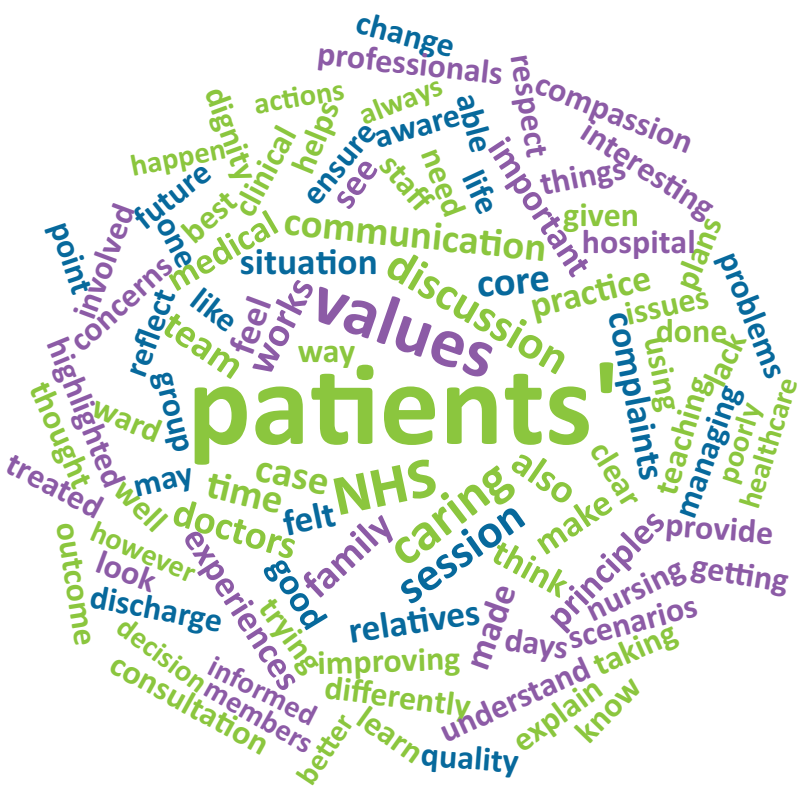

Fig 3. Word cloud of most frequently used words in the submitted reflections.
The themes could be broadly categorised into four main areas: patient-focused themes, NHS value specific themes, personal development themes and themes directly pertaining to the teaching session. These headings will be used to look at the results in more details.

\section{Patient-focused themes}

\section{Theme: Advocating for patients / patient-centred care}

Patients were the focus of the majority of reflections with participants considering how the session had empowered them:

I felt more of an advocate for the patients having completed this session.

And considering how they could practically adopt a more patient-centred approach:

I need to consider ... if this treatment was started, what would I like to know as a patient / family member. Acting appropriately upon this would improve the NHS value of making the patient the centre of care.

Theme: Patient safety

Patient safety was a theme in reflections that related to specific clinical situations with safety issues that could have resulted from both action and inaction by those involved:

One should always remember patient safety and should always make sure that that is priority over not liking his/her team.

This could have potentially impacted on patient safety if I had been [too] nervous to contact the consultant directly.

\section{Theme: Communication}

A recurring theme in the reflections analysed was communication. Some identified this as a learning point from the teaching session:

The key message I took away was the importance of communication with patients and their relatives.

The training sessions led me to reflect on my communication with my patients.

Others reflected on how poor communication with patients can be:

There have been many situations in my work where patients have not been communicated to adequately about their care.

I was shocked and disappointed to realise how poor we as a team were at ensuring patients under our care are fully up-to-date.

Many identified the importance of, or need for, good communication:

I have seen in my practice, that no matter what the outcome, patients and their relatives are generally happier if they are well informed.

Actually taking time out to communicate to the patient and relatives can be just as important as the medical treatment.

Communication was also identified as a contributing factor to how well functioning the patient-doctor team was and how the healthcare team worked together: 
I feel that in most of the cases the doctor's decisions were done in the patient's best interests but the communication was lacking.

We need to be mindful of communication deficiencies and ensure we take the time to relay information to patients and allied health professionals.

While there was a consistent theme of changing practice, many were more specifically focused on communication being by far the most common failing:

Following this session, I now take extra effort to ensure I introduce myself clearly and make sure the patient understands fully what I am explaining.

Pledge to make small and simple changes (ie proper introduction when meeting patients and family members.

\section{Theme: Multiple failings}

The following quotes refer to the complaints that were looked at in the group work section of the teaching session. It was widely recognised that the situations that arose were as a result of multiple failings rather than one catastrophic or single blame event or error:

Complaints were often not of one big event, but of a catalogue of smaller errors or oversights which occurred over the length of the patients stay.

The patients in the case studies all felt as if they had not been cared for appropriately (indeed they hadn't) mostly due to small oversights as opposed to large mistakes.

It highlighted where problems arise and how simple issues can accumulate to create larger issues which result in poor management of patients and the NHS values being lost.

\section{Theme: Small things = big difference}

The converse theme was also identified, highlighting the positive effects of small things:

I have learnt from the session the difference which small amendments can make and the impact this can have on the patient, their health, dignity and wellbeing.

It can be simple things such as cups of tea, patients being able to go to the toilet, clean sheets and taking time to explain things to patients that make a massive difference to a patient's journey.

\section{NHS value-specific themes}

\section{Theme: Dignity}

Some reflections identified situations where specific NHS values were not upheld:

I feel the patient's dignity was probably not respected the way we left things.

In the future I would take the time to ensure that the patient's dignity was the first priority here.

\section{Theme: Compassion/empathy}

The importance of compassion and empathy was recognised:
Compassion costs very little in [terms] of time but does have longlasting impacts on patients.

We should remember that these people are often scared, anxious, homesick and wanting some compassion and security. If we can't provide them that, we can't provide them with much.

Some reflected on more specific situations or scenarios with reference to empathy:

I felt shocked about the lack of empathy of the staff involved.

\section{Theme: Teamwork}

The roles of good and poor teamwork was recognised by participants:

This session has helped me to reflect on the overall teamworking to aid the patient experience.

The manner in which we as a team had conducted ourselves was not up to the standards that are expected and set in the core values of the NHS.

\section{Personal development themes}

\section{Theme: Changing own practice}

Participants frequently recognised areas of their own practice that could be improved or changed. Many of these were generic take home messages:

I will use what I have learnt in my approach to dealing with complaints and difficult situations.

Hold onto values and remember to see everything from the patient's perspective as well as the medical perspective.

I left the session motivated to make a change.

\section{Theme: Raising concerns and empowerment}

Various aspects of raising concerns came through. A large number expressed this as an area to improve in their future practice or identified the responsibility that they themselves have to raise concerns:

When I see something that I feel is not right or inappropriate, I will try to fill in critical incident forms in order to minimise the chance of a similar situation [occurring] again.

In future if there are areas of concern, I now have new skill sets, such as how to and who to approach, to voice my concerns. Will also be more confident in doing this in a more diplomatic manner.

Although the Francis report was unpleasant to hear about, it confirmed that we really have a responsibility to ensure our own hospitals do not get into such situations.

\section{Theme: Juggling and balancing pressures}

Prioritising is a key part of being a foundation doctor, made necessary by the reality and demands of the hospital environment. Workload was among the issues highlighted:

However, we are so busy sometimes to even provide them with just a few reassuring words. 
I feel like you can become alienated from the actions of caring for patients and become more focused on getting all of the little tick-box jobs done.

It makes me sad that I cannot always offer the same level of care to every patient because of staffing, volume of patients, equipment, space to see the patient, time pressures etc.

\section{Theme: Encouraging and improving reflection}

The exercise of filling out the online form on their portfolio was evidence of engagement in reflection as a result of the NHS values teaching session itself, however, many also expressly or subtly highlighted reflection within their reflections:

I felt that for our group it was a poignant experience, we were forced to reflect on our daily practice and really consider whether we were working to the best of our ability for the best of our patients.

The ... NHS values provides a key resource for personal reflection to ensure high quality care is being delivered on a day-to-day basis

It made me reflect on the human aspects of patients rather than the medical/surgical aspects of patients.

This session made me think of my day-to-day practice in a different light. Do I incorporate all these values? What can I do to make my practice better?

\section{Themes directly pertaining to the teaching session}

Theme: Reflection on the impact of the teaching session

The impact of the session on participants was evident in the number of reflections that focused on the teaching session itself:

It has re-ignited my enthusiasm [for] the NHS; a noble project designed to help the poorest of society. I hope to embody the principles and ideals in the NHS in every action I undertake as a doctor.

It was good going through case-based discussions to really highlight how the NHS values can be forgotten but at the same time they are really easy to uphold without it being a burden to us.

I enjoyed the session and I think that it was a good way to cement the NHS core values in the heads of junior doctors.

[While] discussing the cases, I thought that if all healthcare staff were made aware of NHS values and underwent this session, then many adverse events in the cases would not have happened as they did.

Although, several also lamented the necessity of these values being reinforced:

I felt somewhat saddened that we needed a specific talk that highlight the fact that we need, for example, to show patients respect and maintain [their] [dignity] or that we need to improve their lives.

Overall, it was an enjoyable and positive experience, just a shame it has become a necessary experience.

\section{Theme: Content and delivery of teaching session}

A recurring theme was new knowledge of the structure of the NHS and NHS values and constitution. Many expressly stated that they did not have an awareness of the NHS values in advance of the session.

I, like most of the other FY1s, was not aware of the core values. It was something relevant to us and it was good for us to be made aware of these.

I feel that I now have a better understanding of the values of the NHS and their importance. I feel that this is not always addressed in training and we may sometimes forget the common aim that we all have.

It has made me more aware of the greater structure of the NHS and shed new light on the challenges that the organisation faces.

The group work part of the teaching session was particularly well received by participants:

Group work allowed the session to be more interactive and enjoyable. Working in a group enable dynamic discussion.

The complaints that were used as case studies in the teaching session evoked a number of feelings and were readily related to by participants:

Going through this case I did actually feel shocked at the fact that this did really happen.

As doctors, it is easy to become desensitised to the shortcomings of the system and the effect this has on patients but when reading complaints from the relatives' point of [view], this reminds us that we would be just as angry if our own relative received bad care.

When we did the scenarios, I could relate to several elements and had seen similar situations already in the 6 months of working in the NHS.

\section{Theme: Negative feedback, disengagement or apathy}

In a few of the reflections analysed, feedback about the session was less positive:

I did not find the session useful ... I do not feel I need to read a set of values to tell me how to behave in the NHS.

It felt like the 3 hours could have been better spent either doing the job we are paid for or being taught about something more appropriate.

In one case, a participant had been frustrated by the attitude of the group:

Some of the doctors were quite dismissive of the exercise. They stated things like, it was all nursing mistakes, and did not really take the task seriously... I was disappointed with my group and I thought their attitude reflected why we need to have such teaching.

Not all of the submitted reflections engaged with the process. A small number had submitted blank forms, forms with one word in each section or with only one section of the reflective cycle completed.

\section{Discussion}

The results of our thematic analysis show a wide-ranging spectrum of themes within participant reflections. This demonstrates that the session is capable of addressing facets of all the NHS 
values. Evaluating training where values and professionalism is the focus is inherently difficult. Our values as people and doctors are a sum total of our experiences and how we are taught to deal with them, and professionalism is an evolving process over time. ${ }^{9}$ The challenge is to provide opportunities to explore not only what these values are but how they are applicable to daily clinical practice. ${ }^{10}$ The NHS constitution is essential in this sense in providing a consensus and template of what exactly should be taught and it is clear that foundation doctors are not universally aware of the existence of this document and its contents. ${ }^{2}$ Reassuringly, despite this, the analysis showed the clear focus on patients that these professionals have. While it is understandable that some participants felt that values cannot be taught, this is contradicted by the insight provided throughout the reflections into how participants awareness and mindfulness of the application of NHS values to their clinical practice increased.

The reflective form appears to provide focus for the trainees in consolidating their learning from a teaching session that covers such a quantity of material. It also brings the added benefit to participants of being able to map the session to the curriculum easily, as NHS values match well with the professionalism aspects of the foundation curriculum. ${ }^{11}$ It is likely that those who completed the reflective form were more engaged in the process and, as such, these results need to be taken alongside other, compulsory, feedback from the session. However, both the depth and breadth of the reflections analysed indicates the suitability of this type of session for providing an introduction or refresher of the NHS values and their application to clinical practice.

Training in itself is only one aspect of bringing about cultural change within our NHS and we would advocate a situated learning approach for professionalism with integration of values into all aspects of training. ${ }^{12} \mathrm{~A}$ small study of medical students and doctors in England found that learning professionalism through experience is preferred but the knowledge of the values that underpin this professionalism is essential to then develop this through experience and clinical practice. ${ }^{13}$ We have shown that a carefully planned and delivered teaching session can provide this baseline. .

\section{Supplementary material}

Additional supplementary material may be found in the online version of this article at www.rcpjournals.org/fhj: S1 - Example complaint.

\section{References}

1 Francis R (chair). Report of the Mid Staffordshire NHS Foundation Trust Public Inquiry: executive summary. London: The Stationery Office, 2013:66.

2 NHS. The NHS constitution: the NHS belongs to us all. Department of Health, 2013.

3 Squazzo JD. Ethical wisdom. Doing the right thing, every day, everywhere in the organisation. Healthc Exec 2011;26:30-2, 34-5.

4 Martin E (ed). Concise Medical Dictionary, 9th edn. Oxford University Press, 2015:Ethical erosions. http://oxfordindex.oup.com/ view/10.1093/acref/9780199557141.013.12831 [Accessed 15 December 2016].

5 Vetter N. Editorial: Values in the NHS. J Public Health Med 2003; 25:1-3.

6 Department of Health. Hard truths: The journey to putting patients first: Volume one of the government response to the Mid Staffordshire NHS Foundation Trust public inquiry. The Stationery Office, 2014.

7 Department of Health. Culture change in the NHS: Applying the lessons of the Francis Inquiries. The Stationery Office, 2015.

8 Gibbs G. Learning by doing: a guide to teaching and learning methods. Oxford Polytechnic, 1988.

9 Zuckerman JD, Holder JP, Mercurii J], Philips DP, Egol KA. Teaching professionalism in orthopaedic surgery residency programs. J Bone Joint Surg 2012;94:e51.

10 Fish D, Coles C. Medical education: Developing a curriculum for practice. Open University Press, 2007.

11 UK Foundation Programme. The foundation programme curriculum. UK Foundation Programme, 2016. www.foundationprogramme.nhs.uk/pages/home/training-and-assessment [Accessed 16 December 2016].

12 Cruess RL, Cruess SR. Teaching professionalism: general principles. Med Teach 2006;28:205-8.

13 Riley S, Kumar N. Teaching medical professionalism. Clin Med 2012;12:9-11.

Address for correspondence: Dr Linda Watkins, Glasgow Royal Infirmary, 84 Castle Street, Glasgow G2 6QE, UK. Email: linda.watkins@ggc.scot.nhs.uk

Twitter: @RadDrWat 